Review Article

\title{
A Review on Analysis and Synthesis of Nonlinear Stochastic Systems with Randomly Occurring Incomplete Information
}

\author{
Hongli Dong, ${ }^{1,2}$ Zidong Wang, $^{3,4}$ Xuemin Chen, ${ }^{5}$ \\ and Huijun Gao ${ }^{6}$ \\ ${ }^{1}$ College of Electrical and Information Engineering, Northeast Petroleum University, \\ Daqing 163318, China \\ ${ }^{2}$ Institute for Automatic Control and Complex Systems, University of Duisburg-Essen 47057, Germany \\ ${ }^{3}$ Department of Automation, Tsinghua University, Beijing 100084, China \\ ${ }^{4}$ Department of Information Systems and Computing, Brunel University, Uxbridge, Middlesex, \\ UB8 3PH, UK \\ ${ }^{5}$ Department of Engineering Technology, Texas Southern University, 3100 Cleburne Street, Houston, \\ TX 77004, USA \\ ${ }^{6}$ Research Institute of Intelligent Control and Systems, Harbin Institute of Technology, \\ Harbin 150001, China
}

Correspondence should be addressed to Hongli Dong, shiningdhl@gmail.com

Received 11 November 2012; Accepted 21 November 2012

Academic Editor: Bo Shen

Copyright (C 2012 Hongli Dong et al. This is an open access article distributed under the Creative Commons Attribution License, which permits unrestricted use, distribution, and reproduction in any medium, provided the original work is properly cited.

In the context of systems and control, incomplete information refers to a dynamical system in which knowledge about the system states is limited due to the difficulties in modeling complexity in a quantitative way. The well-known types of incomplete information include parameter uncertainties and norm-bounded nonlinearities. Recently, in response to the development of network technologies, the phenomenon of randomly occurring incomplete information has become more and more prevalent. Such a phenomenon typically appears in a networked environment. Examples include, but are not limited to, randomly occurring uncertainties, randomly occurring nonlinearities, randomly occurring saturation, randomly missing measurements and randomly occurring quantization. Randomly occurring incomplete information, if not properly handled, would seriously deteriorate the performance of a control system. In this paper, we aim to survey some recent advances on the analysis and synthesis problems for nonlinear stochastic systems with randomly occurring incomplete information. The developments of the filtering, control and fault detection problems are systematically reviewed. Latest results on analysis and synthesis of nonlinear stochastic systems are discussed in great detail. In addition, various distributed filtering technologies over sensor networks are highlighted. Finally, some concluding remarks are given and some possible future research directions are pointed out. 


\section{Introduction}

In the past decade, networked control systems (NCSs) have attracted much attention owing to their successful applications in a wide range of areas for the advantage of decreasing the hardwiring, the installation cost, and implementation difficulties. Nevertheless, the NCSrelated challenging problems arise inevitably due to the physical equipment constraints and the complexity and uncertainty of the external environment in the process of modeling or information transmission, which would drastically degrade the system performances. Such network-induced problems include, but are not limited to, missing measurements, communication delays, sensor and actuator saturations, signal quantization, and randomly varying nonlinearities. These phenomena may occur in a probabilistic way that are customarily referred to as the randomly occurring incomplete information. Note that, in the literature concerning systems and control, incomplete information usually refers to a dynamical system in which knowledge about the system states is limited due to the difficulties in modeling complexity in a quantitative way. The well-known types of incomplete information include parameter uncertainties and norm-bounded nonlinearities.

For several decades, nonlinear analysis and stochastic analysis are arguably two of the most active research areas in systems and control. This is simply because (1) nonlinear control problems are of interest to engineers, physicists, and mathematicians because most physical systems are inherently nonlinear in nature and (2) stochastic modelling has come to play an important role in many branches of science and industry as many real world systems and natural processes may be subject to stochastic disturbances. There has been rich literature on the general nonlinear stochastic control problems. A great number of techniques have been developed on filtering, control, and fault detection problems for nonlinear stochastic systems in order to meet the needs of practical engineering. Recently, with the development of networked control systems, the analysis and synthesis problems for nonlinear stochastic systems with randomly occurring incomplete information have become interesting and imperative yet challenging topics that have gained a great deal of research attention.

The focus of this paper is to provide a timely review on the recent advances of the analysis and synthesis issues for nonlinear stochastic systems with randomly occurring incomplete information. Most commonly used methods for modeling randomly occurring incomplete information are summarized. Based on the models established, various filtering, control, and fault detection problems with randomly occurring incomplete information are discussed in great detail. Such kind of randomly occurring incomplete information typically appears in a networked environment, which includes randomly occurring uncertainties, randomly occurring nonlinearities, randomly occurring saturation, randomly missing measurements, and randomly occurring quantization. Subsequently, latest results on analysis and synthesis of nonlinear stochastic systems with randomly occurring incomplete information are reviewed. Finally, some concluding remarks are drawn and some possible future research directions are pointed out.

The rest of this paper is outlined as follows. In Section 2, the phenomenon of randomly occurring incomplete information is addressed and the corresponding models are summarized. In Section 3, the analysis and synthesis problems for nonlinear stochastic systems are reviewed. Section 4 discusses the distributed filtering problems over sensor networks. The latest results on filtering, control, and fault detection problems for nonlinear stochastic systems with randomly occurring incomplete information are reviewed in Section 5. In Section 6, we give some concluding remarks and also point out some future directions. 


\section{Randomly Occurring Incomplete Information}

Accompanied by the rapid development of communication and computer technology, NCSs have become more and more popular for their successful applications in modern complicated industry processes, for example, aircraft and space shuttle, nuclear power stations and high-performance automobiles. However, the insertion of network makes the analysis and synthesis problems much more complex due to the randomly occurring incomplete information that is mainly caused by the limited bandwidth of the digital communication channel. The randomly occurring incomplete information under consideration mainly includes missing measurements, communication delays, sensor and actuator saturations, signal quantization, and randomly varying nonlinearities.

\subsection{Missing Measurements}

In practical systems within a networked environment, the measurement signals are usually subject to probabilistic information missing (data dropouts or packet losses), which may be caused for a variety of reasons, such as the high manoeuvrability of the tracked target, a fault in the measurement, intermittent sensor failures, network congestion, accidental loss of some collected data, or some of the data may be jammed or coming from a very noisy environment, and so forth. Such a missing measurement phenomenon that typically occurs in networked control systems has attracted considerable attention during the past few years, see [1-11] and the references therein. Various approaches have been presented in the literature to model the packet-dropout phenomenon. For example, in [12,13], the data packetdropout phenomenon has been described as a binary switching sequence that is specified by a conditional probability distribution taking on values of 0 and 1 . In [14], a discrete-time linear system with Markovian jumping parameters has been employed to construct the random packet-dropout model. In [15], a model that comprises former measurement information of the process output has been introduced to account for the successive packet dropout phenomenon. A model of multiple missing measurements has been proposed in $[11,16]$ by using a diagonal matrix to describe the different missing probabilities for individual sensors.

\subsection{Communication Delays}

Owing to the fact that time delays commonly reside in practical systems and constitute a main source for system performance degradation or even instability, the past decade has witnessed a significant progress on analysis and synthesis for systems with various types of delays, and a large amount of literature has appeared on the general topic of time-delay systems. For example, in [17], the stability of NCSs under the network-induced delay has been studied by using a hybrid system technique. The optimal stochastic control method has been proposed in [18] to control the communication delays in NCSs. A networked controller has been designed in the frequency domain using the robust control theory in [19], in which the network delays are considered as an uncertainty. However, most of the relevant literature mentioned above has focused on the constant time delays. Delays resulting from network transmissions are inherently random and time varying [2022]. This is particularly true when signals are transmitted over the internet and therefore existing control methods for constant time delay cannot be directly utilized [23]. Recently, some researchers have started to model the network-induced time delays in multiform 
probabilistic ways and, accordingly, some initial results have been reported. For example, in $[24,25]$, the random communication delays have been modeled as Markov's chains and the resulting closed-loop systems have been represented as Markovian jump linear systems with two jumping parameters. In [26], two kinds of random delays, which happen in the channels from the controller to the plant and from the sensor to the controller, have been simultaneously considered. The random delays have been modeled in [26] as a linear function of the stochastic variable satisfying Bernoulli random binary distribution. Different from [26], the problem of stability analysis and stabilization control design has been studied in [27] for the Takagi-Sugeno (T-S) fuzzy systems with a probabilistic interval delay, and the Bernoulli distributed sequence has been utilized to describe the probability distribution of the time-varying delay taking values in an interval. It should be mentioned that, among others, the binary representation of the random delays has been fairly popular because of its practicality and simplicity in describing communication delays.

However, most research attention has been centered on the single random delay having a fixed value if it occurs. This would lead to conservative results or even degradation of the system performance since, at a certain time, the NCSs could give rise to multiple time-varying delays but with different occurrence probabilities. Therefore, a more advanced methodology is needed to handle time varying network-induced time-delays in a closed-loop control system.

\subsection{Signal Quantization}

As is well-known, quantization always exists in computer-based control systems employing finite-precision arithmetic. Moreover, the performance of NCSs will be inevitably subject to the effect of quantization error owing to the limited network bandwidth caused possibly by strong signal attenuation and perturbation in the operational environment. Hence, the quantization problem of NCSs has long been studied and many important results have been reported in [28-35] and the references therein. For example, in [36], the time-varying quantization strategy has been firstly proposed where the number of quantization levels is fixed and finite while at the same time the quantization resolution can be manipulated over time. In [37], the problem of input-to-state stable with respect to the quantization error for nonlinear continuous-time systems has been studied. In this framework, the effect of quantization is treated as an additional disturbance whose effect is overcome by a Lyapunov redesign of the control law. In [38], a switching control strategy with dwell time has been proposed to use a quantizer for single-input systems. The quantizer employed in this framework is in fact an extension of the static logarithmic quantizer in [39] to continuous case. So far, there have been mainly two different types of quantized communication models adopted in the literature: uniform quantization [28, 33, 34] and logarithmic quantization $[30-32,35]$. It has been proved that, as compared to the uniform quantizer, the logarithmic quantization is more preferable since fewer bits need to be communicated. In [40], a sector bound scheme has been proposed to handle the logarithmic quantization effects in feedback control systems, and such an elegant scheme has then been extensively employed later on, see, for example $[7,35,41,42]$, and the references therein. However, we note that the methods that most of the references cited above could not be directly applied to NCSs, because in NCSs the effects of network-included delay and packet dropout should be also considered. 


\subsection{Sensor and Actuator Saturations}

In practical control systems, sensors and actuators cannot provide unlimited amplitude signal due primarily to the physical, safety, or technological constraints. In fact, the actuator/sensor saturation is probably the most common nonlinearity encountered in practical control systems, which can degrade the system performance or even cause instability if such a nonlinearity is ignored in the controller/filter design. Because of their theoretical significance and practical importance, considerable attention has been focused on the filtering and control problems for systems with actuator saturation [43-46]. As for sensor saturation, the associated results have been relatively few due probably to the technical difficulty [47-49]. Nevertheless, in the scattered literature regarding sensor saturation, it has been implicitly assumed that the occurrence of sensor saturations is deterministic, that is, the sensor always undergoes saturation. Such an assumption, however, does have its limitation especially in a sensor network. The sensor saturations may occur in a probabilistic way and are randomly changeable in terms of their types and/or levels due to the random occurrence of networkinduced phenomena such as random sensor failures, sensor aging, or sudden environment changes. To reflect the reality in networked sensors, in [8], a new phenomenon of sensor saturation, namely, randomly occurring sensor saturation (ROSS), has been put forward in order to better reflect the reality in a networked environment. A novel sensor model has then been established to account for both the ROSS and missing measurement in a unified representation by using two sets of the Bernoulli distributed white sequences with known conditional probabilities. It should be mentioned that very few results have dealt with the systems with simultaneous presence of actuator and sensor saturations [50] although such a presence is quite typical in engineering practice.

\subsection{Randomly Varying Nonlinearities}

It is well known that nonlinearities exist universally in practice and it is quite common to describe them as additive nonlinear disturbances that are caused by environmental circumstances. In a networked system such as the internet-based three-tank system for leakage fault diagnosis, such nonlinear disturbances may occur in a probabilistic way due to the random occurrence of network-induced phenomenon. For example, in a particular moment, the transmission channel for a large amount of packets may encounters severe network-induced congestions due to the bandwidth limitations, and the resulting phenomenon could be reflected by certain randomly occurring nonlinearities where the occurrence probability can be estimated via statistical tests. As discussed in [51, 52], in nowadays prevalent networked control system, the nonlinear disturbances themselves may experience random abrupt changes due to random changes and failures arising from network-induced phenomenon, which give rise to the so-called randomly varying nonlinearities. In other words, the type and intensity of the so-called randomly varying nonlinearities could be changeable in a probabilistic way.

\section{The Analysis and Synthesis of Nonlinear Stochastic Systems}

For several decades, stochastic systems have received considerable research attention in which stochastic differential equations are the most useful stochastic models with broad applications in aircraft, chemical or process control system, and distributed networks. 
Generally speaking, stochastic systems can be categorized into two types, namely, internal stochastic systems and external stochastic systems [53].

As a class of internal stochastic systems with finite operation modes, the Markovian jump systems (MJSs) have gained particular research interests in the past two decades because of their practical applications in a variety of areas such as power systems, control systems of a solar thermal central receiver, networked control systems, manufacturing systems, and financial markets. So far, existing results about MJSs have covered a wide range of research problems including those for stability analysis [54-56], filter design [57-64], and controller design [65, 66]. Nevertheless, compared to the fruitful results for filtering and control problems of MJSs, the corresponding fault detection problem of MJSs has received much less attention $[67,68]$ due primarily to the difficulty in accommodating the multiple fault detection performances. In the literature concerning the MJSs, most results have been reported by supposing that the transition probabilities (TPs) in the jumping process are completely accessible. However, this is not always true for many practical systems. For example, in networked control systems, it would be extremely difficult to obtain precisely all the TPs via time-consuming yet expensive statistical tests. In other words, some of TPs are very likely to be incomplete (i.e., uncertain or even unknown). So far, some initial efforts have been made to address the incomplete probability issue for MJSs. For example, the problems of uncertain TPs and partially unknown TPs have been addressed in [56, 62] and [63, 69], respectively. Furthermore, the concept of deficient statistics for modes transitions has been put forward in [70] to reflect different levels of the limitations in acquiring accurate TPs. Unfortunately, up to now, the filtering/control/fault detection problem for discrete-time Markovian jump systems with randomly varying nonlinearities has not been fully investigated yet.

For external stochastic systems, stochasticity is always caused by external stochastic noise signal and can be modelled by stochastic differential equations with stochastic processes [53]. Furthermore, recognizing that nonlinearities exist universally in practice and both nonlinearity and stochasticity are commonly encountered in engineering practice, the robust $H_{\infty}$ filtering, $H_{\infty}$ control, and fault detection problems for nonlinear stochastic systems have stirred a great deal of research interests. For the fault detection problems, we refer the readers to $[46,71-73]$ and the references therein. With respect to the $H_{\infty}$ control and filtering problems, we mention some representative work as follows. The stochastic $H_{\infty}$ filtering problem for time-delay systems subject to sensor nonlinearities have been dealt with in [74, 75]. The robust stability and controller design problems for networked control systems with uncertain parameters have been studied in [25, 76], respectively. The stability issue has been addressed in [77] for a class of T-S fuzzy dynamical systems with time delays and uncertain parameters. In [78], the robust $H_{\infty}$ filtering problem for affine nonlinear stochastic systems with state and external disturbance-dependent noise has been studied, where the filter can be designed by solving second-order nonlinear Hamilton-Jacobi inequalities. So far, in comparison with the fruitful literature available for continuous-time systems, the corresponding $H_{\infty}$ filtering results for discrete-time systems have been relatively few. Also, to the best of our knowledge, the analysis and design problems for nonlinear discrete-time stochastic systems with randomly occurring incomplete information have not been properly investigated yet, which still remain as challenging research topics.

\section{Distributed Filtering over Sensor Networks}

In the past decade, sensor networks have been attracting increasing attention from many researchers in different disciplines owing to the extensive applications of sensor networks 
in many areas including surveillance, environment monitoring, information collection, industrial automation, and wireless networks [79-88]. A sensor network typically consists of a large number of sensor nodes and also a few control nodes, all of which are distributed over a spatial region. The distributed filtering or estimation, as an important issue for sensor networks, has been an area of active research for many years. Different from the traditional filtering for a single sensor [58,61,89], the information available for the filter algorithm on an individual node of the sensor network is not only from its own measurement but also from its neighboring sensors' measurements according to the given topology. As such, the objective of filtering based on a sensor network can be achieved in a distributed yet collaborative way. It is noticed that one of the main challenges for distributed filtering lies in how to handle the complicated coupling issues between one sensor and its neighboring sensors.

In recent years, the distributed filtering problem for sensor networks has received considerable research interest and a lot of research results have been available in the literature, see, for example, [79, 82, 83, 87, 90-93]. The distributed diffusion filtering strategy has been established in $[79,90]$ for the design of distributed Kalman filters and smoothers, where the information is diffused across the network through a sequence of Kalman iterations and data-aggregation. A distributed Kalman Filtering (DKF) algorithm has been introduced in [93] through which a crucial part of the solution is used to estimate the average of $n$ signals in a distributed way. Furthermore, three novel distributed Kalman filtering algorithms have been introduced in [92] with the first one being a modification of the previous DKF algorithm [93]. Also, a continuous-time DKF algorithm has been rigorously derived and analyzed in [92], and the corresponding extension to the discrete-time setting has been conducted in [83] which includes the optimality and stability analysis.

It should be pointed out that, so far, most reported distributed filter algorithms for sensor networks have been mainly based on the traditional Kalman filtering theory that requires exact information about the plant model. In the presence of unavoidable parameter drifts and external disturbances, a desired distributed filtering scheme should be made as robust as possible. However, the robust performance of the available distributed filters has not yet been thoroughly studied, and this would inevitably restrict the application potential in practical engineering. Therefore, it is of great significance to introduce the $H_{\infty}$ performance requirement with the hope to enhance the disturbance rejection attenuation level of designed distributed filters. Note that some initial efforts have been made to address the robustness issue. Very recently, a new distributed $H_{\infty}$-consensus performance has been proposed in [86] to quantify the consensus degree over a finite horizon and the distributed filtering problem has been addressed for a class of linear time-varying systems in the sensor network, and the filter parameters have been designed recursively by resorting to the difference linear matrix inequalities. In [94], an $H_{\infty}$-type performance measure of disagreement between adjacent nodes of the network has been included and a robust filtering approach has been proposed to design the distributed filters for uncertain plants. On the other hand, since nonlinearities are ubiquitous in practice, it is necessary to consider the distributed filtering problem for target plants described by nonlinear systems.

Unfortunately, up to now, the distributed nonlinear $H_{\infty}$ filtering problem for sensor networks has gained very little research attention despite its practical importance.

\section{Latest Progress}

In $[95,96]$, the $H_{\infty}$ filtering and control problems have been investigated for systems with repeated scalar nonlinearities and missing measurements. The nonlinear system is described 
by a discrete-time state equation involving a repeated scalar nonlinearity which typically appears in recurrent neural networks. The $H_{\infty}$ filtering problem has been first considered in [95] for the systems with missing measurements. The missing measurements have been modeled by a stochastic variable satisfying the Bernoulli random binary distribution. The quadratic Lyapunov function has been used to design both full- and reduced-order $H_{\infty}$ filters such that, for the admissible random measurement missing and repeated scalar nonlinearities, the filtering error system is stochastically stable and preserves a guaranteed $H_{\infty}$ performance. In addition, in [96], the notion of missing measurements has been extended to the multiple missing measurements, where the missing probability for each sensor is governed by an individual random variable satisfying a certain probabilistic distribution in the interval $[0,1]$. An observer-based feedback controller has been designed to stochastically stabilize the networked system. Both the stability analysis and controller synthesis problems have been investigated in detail.

In [97], the robust $H_{\infty}$ filtering problem has been studied for a class of uncertain nonlinear networked systems with both multiple stochastic time-varying communication delays and multiple packet dropouts. The missing measurements have been modeled via a diagonal matrix consisting of a series of mutually independent random variables satisfying certain probabilistic distributions on the interval $[0,1]$. Such a modeling approach can describe the following packet dropouts situations for the measurement signals: completely missing, completely available, partially missing, and the case when the individual sensor has different missing probability is also included. A new model has been proposed to account for the randomly occurring communication delays. Furthermore, the discrete-time system under consideration has been also subject to parameter uncertainties, state-dependent stochastic disturbances, and sector-bounded nonlinearities. By constructing new Lyapunov functionals, intensive stochastic analysis has been carried out to obtain the desired robust $H_{\infty}$ filter parameters. Furthermore, in [98], by using similar analysis techniques, some parallel results have been extended to the robust $H_{\infty}$ fuzzy output feedback control problem for a class of uncertain discrete-time fuzzy systems with both multiple probabilistic delays and multiple missing measurements.

Considering the case that the transfer function method cannot effectively deal with the nonlinear time-varying systems, a recursive matrix inequalities technique has been proposed in [16] in time domain to deal with the robust finite-horizon filtering problem for a class of uncertain nonlinear discrete time-varying stochastic systems with multiple missing measurements and error variance constraints. All the system parameters are time varying and the uncertainty enters into the state matrix. By developing a new filter design algorithm for finite-horizon case, sufficient conditions have been derived for a finite-horizon filter to satisfy the estimation error variance constraints, robustness, and the prescribed $H_{\infty}$ performance requirement. A simulation example about the target tracking problem has demonstrated the effectiveness and practicality of the developed filter design scheme. This paper has addressed the open finite-horizon filtering problem satisfying multiple performance indices for a class of uncertain nonlinear discrete time-varying stochastic systems with limited communication. Moreover, by using similar analysis techniques, some parallel results have also been derived in [99] for the corresponding robust $H_{\infty}$ finite-horizon output feedback control problem with both sensor and actuator saturations. The obtained results have practical meaning for the tracking problem of highly maneuvering targets.

In $[57,100]$, the filtering and fault detection problems have been investigated for discrete-time Markovian jump systems with randomly varying nonlinearities (RVNs) and sensor saturation. The issue of RVNs has been first addressed and the considered transition 
probability matrix includes the case with polytopic uncertainties and the case with partially unknown transition probabilities, respectively. The $H_{\infty}$ filtering problem has been first considered in [57], where the randomly occurring nonlinearities have been modeled by the Bernoulli distributed white sequences with known conditional probabilities. Sufficient conditions have been derived for the filtering augmented system under consideration to satisfy the $H_{\infty}$ performance constraint. The corresponding robust $H_{\infty}$ filters have been designed by solving sets of recursive linear matrix inequalities. Based on this, the corresponding fault detection filter design problem has been studied in [100]. Two energy norm indices have been utilized for the fault detection problem in order to account for, respectively, the restraint of disturbance and the sensitivity of faults. A locally optimized fault detection filter has been designed by developing a novel algorithm such that the effect from the exogenous disturbance on the residual is attenuated with respect to a minimized $H_{\infty}$ norm, and the sensitivity of the residual to the fault is enhanced in terms of a maximized $H_{\infty}$ norm.

By noticing that the aforementioned scheme cannot be applied to complex dynamic systems with transmission delay or state delay, in [41, 101], the fault detection problems have been dealt with for two classes of discrete-time nonlinear mixed stochastic time delay systems with limited communication. The mixed time delays involve both the multiple timevarying discrete delays and the infinite distributed delays. The fault detection problem has been first addressed in [41] for a class of discrete-time systems with randomly occurring nonlinearities and mixed stochastic time delays as well as measurement quantizations. Sufficient conditions have been established via intensive stochastic analysis for the existence of the desired fault detection filters, and then the explicit expression of the desired filter gains has been derived by means of the feasibility of certain matrix inequalities. Moreover, in [101], the developed scheme has been extended to the robust fault detection problem for a class of uncertain discrete-time T-S fuzzy systems with stochastic mixed time delays and successive packet dropouts. Two practical examples have been provided to show the usefulness and effectiveness of the proposed design methods.

Considering the case that the occurrence of incomplete information in sensor network is more complex and severer, the studies in [102-104] have investigated the distributed filtering problem for several classes of nonlinear stochastic systems over lossy sensor networks. The issues of average $H_{\infty}$ performance constraints have been brought up in [102], and then the distributed $H_{\infty}$ filtering problem has been investigated for system with repeated scalar nonlinearities and multiple probabilistic packet losses. Moreover, in [103], the distributed filtering problem has been further extended to the nonlinear time-varying systems with limited communication. The time-varying system (target plant) is subject to randomly vary nonlinearities caused by environmental circumstances. The lossy sensor network suffers from quantization errors and successive packet dropouts that are described in a unified framework. A new distributed finite-horizon filtering technique by means of a set of recursive linear matrix inequalities has been proposed to satisfy the prescribed average filtering performance constraint. In addition, the distributed $H_{\infty}$ filtering problem has been investigated in [104] for a class of discrete-time Markovian jump nonlinear timedelay systems with deficient statistics of mode transitions. A novel model that describes the deficient statistics of modes transitions has been proposed to account for known, bounded uncertain, and unknown transition probabilities. The system measurements have been collected through a lossy sensor network subject to randomly occurring quantizations errors (ROQEs) and randomly occurring packet dropouts (ROPDs). Two sets of the Bernoulli distributed white sequences have been introduced to govern the phenomena of ROQEs and 
ROPDs in the lossy sensor network. The system model (dynamical plant) includes the modedependent Lipschitz-like nonlinearities. The distributed filters have been designed to obtain sufficient conditions for ensuring stochastic stability as well as the prescribed average $H_{\infty}$ performance constraint.

In [105], a new approach has been proposed in virtue of the solvability of certain coupled recursive Riccati difference equations (RDEs) to deal with the distributed $H_{\infty}$ state estimation problem for a class of discrete time-varying nonlinear systems with both stochastic parameters and stochastic nonlinearities. By employing the completing squares method and the stochastic analysis technique, a necessary and sufficient condition has been established to ensure the dynamics of the estimation error to satisfy the $H_{\infty}$ performance constraint. Furthermore, the estimator gains have been explicitly characterized by means of the solutions to two coupled backward recursive RDEs. Finally, an illustrative example has been provided that highlights the usefulness of the developed state estimation approach.

\section{Conclusions and Future Work}

In this paper, we have reviewed some recent advances on the analysis and synthesis problems for nonlinear stochastic systems with randomly occurring incomplete information. Most commonly used randomly occurring incomplete information models have been summarized. Based on this, various filtering, control, and fault detection problems have been discussed. In addition, the various distributed filtering technologies over sensor networks have been given. Latest results on analysis and synthesis problems for nonlinear stochastic systems with randomly occurring incomplete information have been surveyed. Based on the literature review, some related topics for the future research work are listed as follows.

(1) The nonlinearities considered in the existing results have been assumed to satisfy certain constraints for the purpose of simplifying the analysis, thereby bringing a great deal of conservatism. It would be a promising research topic to analyze and synthesize the general nonlinear systems with randomly occurring incomplete information.

(2) Another future research direction is to further investigate multiobjective $H_{2} / H_{\infty}$ control and filtering problems for nonlinear systems with randomly occurring incomplete information.

(3) It would be interesting to investigate the problems of fault detection and fault tolerant control for time-varying systems with randomly occurring incomplete information over a finite time horizon.

(4) A trend for future research is to generalize the methods obtained in the existing results to the control, synchronization, and filtering problems for nonlinear stochastic complex networks systems with randomly occurring incomplete information.

(5) A practical engineering application of the existing theories and methodologies would be fault detection for petroleum well systems.

\section{Acknowledgments}

This work was supported in part by the National Natural Science Foundation of China under Grants 61273156, 61134009, 61273201, 61021002, and 61004067, the Engineering and Physical 
Sciences Research Council (EPSRC) of the UK under Grant GR/S27658/01, the Royal Society of the UK, the National Science Foundation of the USA under Grant No. HRD-1137732, and the Alexander von Humboldt Foundation of Germany.

\section{References}

[1] G. Chen, "Simple treatment for suboptimal Kalman filtering in case of measurement data missing," IEEE Transactions on Aerospace and Electronic Systems, vol. 26, no. 2, pp. 413-415, 1990.

[2] F. O. Hounkpevi and E. E. Yaz, "Robust minimum variance linear state estimators for multiple sensors with different failure rates," Automatica, vol. 43, no. 7, pp. 1274-1280, 2007.

[3] S. Nakamori, R. Caballero-Águila, A. Hermoso-Carazo, J. D. Jiménez-López, and J. Linares-Pérez, "Signal estimation with nonlinear uncertain observations using covariance information," Journal of Statistical Computation and Simulation, vol. 79, no. 1-2, pp. 55-66, 2009.

[4] B. Shen, Z. Wang, H. Shu, and G. Wei, “On nonlinear $H_{\infty}$ filtering for discrete-time stochastic systems with missing measurements," IEEE Transactions on Automatic Control, vol. 53, no. 9, pp. 2170-2180, 2008.

[5] B. Shen, Z. Wang, H. Shu, and G. Wei, " $H_{\infty}$ filtering for uncertain time-varying systems with multiple randomly occurred nonlinearities and successive packet dropouts," International Journal of Robust and Nonlinear Control, vol. 21, no. 14, pp. 1693-1709, 2011.

[6] S. Sun, L. Xie, W. Xiao, and Y. C. Soh, "Optimal linear estimation for systems with multiple packet dropouts," Automatica, vol. 44, no. 5, pp. 1333-1342, 2008.

[7] Z. Wang, B. Shen, H. Shu, and G. Wei, "Quantized $H_{\infty}$ control for nonlinear stochastic time-delay systems with missing measurements," IEEE Transactions on Automatic Control, vol. 57, no. 6, pp. 14311444, 2012.

[8] Z. Wang, B. Shen, and X. Liu, " $H_{\infty}$ filtering with randomly occurring sensor saturations and missing measurements," Automatica, vol. 48, no. 3, pp. 556-562, 2012.

[9] Z. Wang, F. Yang, D. W. C. Ho, and X. Liu, "Robust $H_{\infty}$ filtering for stochastic time-delay systems with missing measurements," IEEE Transactions on Signal Processing, vol. 54, no. 7, pp. 2579-2587, 2006.

[10] Z. Wang, F. Yang, D. W. C. Ho, and X. Liu, "Robust $H_{\infty}$ control for networked systems with random packet losses," IEEE Transactions on Systems, Man, and Cybernetics B, vol. 37, no. 4, pp. 916-924, 2007.

[11] G. Wei, Z. Wang, and H. Shu, "Robust filtering with stochastic nonlinearities and multiple missing measurements," Automatica, vol. 45, no. 3, pp. 836-841, 2009.

[12] H. Gao, Y. Zhao, J. Lam, and K. Chen, " $H_{\infty}$ fuzzy filtering of nonlinear systems with intermittent measurements," IEEE Transactions on Fuzzy Systems, vol. 17, no. 2, pp. 291-300, 2009.

[13] Z. Wang, D. W. C. Ho, Y. Liu, and X. Liu, "Robust $H_{\infty}$ control for a class of nonlinear discrete timedelay stochastic systems with missing measurements," Automatica, vol. 45, no. 3, pp. 684-691, 2009.

[14] Z. Shu, J. Lam, and J. Xiong, "Non-fragile exponential stability assignment of discrete-time linear systems with missing data in actuators," IEEE Transactions on Automatic Control, vol. 54, no. 3, pp. 625-630, 2009.

[15] M. Sahebsara, T. Chen, and S. L. Shah, "Optimal $H_{2}$ filtering with random sensor delay, multiple packet dropout and uncertain observations," International Journal of Control, vol. 80, no. 2, pp. 292301, 2007.

[16] H. Dong, Z. Wang, D. W. C. Ho, and H. Gao, "Variance-constrained $H_{\infty}$ filtering for a class of nonlinear time-varying systems with multiple missing measurements: the finite-horizon case," IEEE Transactions on Signal Processing, vol. 58, no. 5, pp. 2534-2543, 2010.

[17] W. Zhang, M. S. Branicky, and S. M. Phillips, "Stability of networked control systems," IEEE Control Systems Magazine, vol. 21, no. 1, pp. 84-99, 2001.

[18] J. Nilsson, Real-time control systems with delays [Ph.D. dissertation], Lund Institute of Technology, Lund, Sweden, 1998.

[19] F. Gokas, Distributed control of systems over communication networks [Ph.D. dissertation], University of Pennsylvania, Philadelphia, Pa, USA, 2000.

[20] H. Gao and X. Li, " $H_{\infty}$ filtering for discrete-time state-delayed systems with finite frequency specifications," IEEE Transactions on Automatic Control, vol. 56, no. 12, pp. 2935-2941, 2011.

[21] H. Gao, T. Chen, and J. Lam, "A new delay system approach to network-based control," Automatica, vol. 44, no. 1, pp. 39-52, 2008. 
[22] Z. Wang and D. W. C. Ho, "Filtering on nonlinear time-delay stochastic systems," Automatica, vol. 39, no. 1, pp. 101-108, 2006.

[23] B. Chen and X. Liu, "Delay-dependent robust $H_{\infty}$ control for T-S fuzzy systems with time delay," IEEE Transactions on Fuzzy Systems, vol. 13, no. 4, pp. 544-556, 2005.

[24] M. Liu, D. W. C. Ho, and Y. Niu, "Stabilization of Markovian jump linear system over networks with random communication delay," Automatica, vol. 45, no. 2, pp. 416-421, 2009.

[25] H. Zhang, M. Li, J. Yang, and D. Yang, "Fuzzy model-based robust networked control for a class of nonlinear systems," IEEE Transactions on Systems, Man, and Cybernetics A, vol. 39, no. 2, pp. 437-447, 2009.

[26] F. Yang, Z. Wang, Y. S. Hung, and M. Gani, " $H_{\infty}$ control for networked systems with random communication delays," IEEE Transactions on Automatic Control, vol. 51, no. 3, pp. 511-518, 2006.

[27] D. Yue, E. Tian, Y. Zhang, and C. Peng, "Delay-distribution-dependent stability and stabilization of T-S fuzzy systems with probabilistic interval delay," IEEE Transactions on Systems, Man, and Cybernetics B, vol. 39, no. 2, pp. 503-516, 2009.

[28] D. V. Dimarogonas and K. H. Johansson, "Quantized agreement under time-varying communication topology," in American Control Conference (ACC '08), pp. 4376-4381, Seattle, Wash, USA, June 2008.

[29] H. Gao and T. Chen, "A new approach to quantized feedback control systems," Automatica, vol. 44, no. 2, pp. 534-542, 2008.

[30] Z. Gao and S. X. Ding, "Actuator fault robust estimation and fault-tolerant control for a class of nonlinear descriptor systems," Automatica, vol. 43, no. 5, pp. 912-920, 2007.

[31] H. R. Karimi, "Robust $H_{\infty}$ filter design for uncertain linear systems over network with networkinduced delays and output quantization," Modeling, Identification and Control, vol. 30, no. 1, pp. 27-37, 2009.

[32] T. Nakamura, "Development of self-learning vision-based mobile robots for acquiring soccer robots behaviors," in Proceedings of the IEEE International Conference on Robotics and Automation. Part 1 (of 4), pp. 2592-2598, Leuven, Belgium, May 1998.

[33] H. Sun, N. Hovakimyan, and T. Basar, " $L_{1}$ adaptive controller for quantized systems," in Proceedings of the American Control Conference, pp. 582-587, San Francisco, Calif, USA, 2011.

[34] C. Y. Tsai and K. T. Song, "Visual tracking control of a wheeled mobile robot with system model and velocity quantization robustness," IEEE Transactions on Control Systems Technology, vol. 17, no. 3, pp. 520-527, 2009.

[35] N. Xiao, L. Xie, and M. Fu, "Stabilization of Markov jump linear systems using quantized state feedback," Automatica, vol. 46, no. 10, pp. 1696-1702, 2010.

[36] R. W. Brockett and D. Liberzon, "Quantized feedback stabilization of linear systems," IEEE Transactions on Automatic Control, vol. 45, no. 7, pp. 1279-1289, 2000.

[37] D. Liberzon, "On stabilization of linear systems with limited information," IEEE Transactions on Automatic Control, vol. 48, no. 2, pp. 304-307, 2003.

[38] H. Ishii and B. A. Francis, "Stabilizing a linear system by switching control with dwell time," IEEE Transactions on Automatic Control, vol. 47, no. 12, pp. 1962-1973, 2002.

[39] N. Elia and S. K. Mitter, "Stabilization of linear systems with limited information," IEEE Transactions on Automatic Control, vol. 46, no. 9, pp. 1384-1400, 2001.

[40] M. Fu and L. Xie, "The sector bound approach to quantized feedback control," IEEE Transactions on Automatic Control, vol. 50, no. 11, pp. 1698-1711, 2005.

[41] H. Dong, Z. Wang, and H. Gao, "On design of quantized fault detection filters with randomly occurring nonlinearities and mixed time-delays," Signal Processing, vol. 92, no. 4, pp. 1117-1125, 2012.

[42] M. Fu and C. E. de Souza, "State estimation for linear discrete-time systems using quantized measurements," Automatica, vol. 45, no. 12, pp. 2937-2945, 2009.

[43] H. Fang, Z. Lin, and T. Hu, "Analysis of linear systems in the presence of actuator saturation and $L_{2}$-disturbances," Automatica, vol. 40, no. 7, pp. 1229-1238, 2004.

[44] T. Hu and Z. Lin, Control Systems With Actuator Saturation: Analysis and Design, Birkäuser, Boston, Mass, USA, 2001.

[45] L. Lv and Z. Lin, "Analysis and design of singular linear systems under actuator saturation and $L_{2} / L_{\infty}$ disturbances," Systems and Control Letters, vol. 57, no. 11, pp. 904-912, 2008.

[46] L. Wu and D. W. C. Ho, "Fuzzy filter design for Itô stochastic systems with application to sensor fault detection," IEEE Transactions on Fuzzy Systems, vol. 17, no. 1, pp. 233-242, 2009.

[47] Y.-Y. Cao, Z. Lin, and B. M. Chen, "An output feedback $H_{\infty}$ controller design for linear systems subject to sensor nonlinearities," IEEE Transactions on Circuits and Systems I, vol. 50, no. 7, pp. 914 921, 2003. 
[48] Y. Xiao, Y.-Y. Cao, and Z. Lin, "Robust filtering for discrete-time systems with saturation and its application to transmultiplexers," IEEE Transactions on Signal Processing, vol. 52, no. 5, pp. 1266-1277, 2004.

[49] Z. Zuo, J. Wang, and L. Huang, "Output feedback $H_{\infty}$ controller design for linear discrete-time systems with sensor nonlinearities," IEE Proceedings, vol. 152, no. 1, pp. 19-26, 2005.

[50] G. Garcia, S. Tarbouriech, and J. M. G. da Silva Jr., "Dynamic output controller design for linear systems with actuator and sensor saturation," in Proceedings of the American Control Conference (ACC '07), pp. 5834-5839, July 2007.

[51] J. Liang, Z. Wang, and X. Liu, “Distributed state estimation for discrete-time sensor networks with randomly varying nonlinearities and missing measurements," IEEE Transactions on Neural Networks, vol. 22, no. 3, pp. 486-496, 2011.

[52] Z. Wang, Y. Wang, and Y. Liu, "Global synchronization for discrete-time stochastic complex networks with randomly occurred nonlinearities and mixed time delays," IEEE Transactions on Neural Networks, vol. 21, no. 1, pp. 11-25, 2010.

[53] M. Liu, Network-based stability analysis and synthesis for stochastic system with Markovian switching [Ph.D. dissertation], City University of Hong Kong, Hong Kong, 2009.

[54] Z. Fei, H. Gao, and P. Shi, "New results on stabilization of Markovian jump systems with time delay," Automatica, vol. 45, no. 10, pp. 2300-2306, 2009.

[55] H. Gao, Z. Fei, J. Lam, and B. Du, "Further results on exponential estimates of Markovian jump systems with mode-dependent time-varying delays," IEEE Transactions on Automatic Control, vol. 56, no. 1, pp. 223-229, 2011.

[56] J. Xiong, J. Lam, H. Gao, and D. W. C. Ho, “On robust stabilization of Markovian jump systems with uncertain switching probabilities," Automatica, vol. 41, no. 5, pp. 897-903, 2005.

[57] H. Dong, Z. Wang, D. W. C. Ho, and H. Gao, "Robust $H_{\infty}$ filtering for Markovian jump systems with randomly occurring nonlinearities and sensor saturation: the finite-horizon case," IEEE Transactions on Signal Processing, vol. 59, no. 7, pp. 3048-3057, 2011.

[58] H. Liu, F. Sun, K. He, and Z. Sun, “Design of reduced-order $H_{\infty}$ filter for Markovian jumping systems with time delay," IEEE Transactions on Circuits and Systems II, vol. 51, no. 11, pp. 1837-1837, 2004.

[59] L. Ma, F. Da, and K.-J. Zhang, "Exponential $H_{\infty}$ filter design for discrete time-delay stochastic systems with Markovian jump parameters and missing measurements," IEEE Transactions on Circuits and Systems I, vol. 58, no. 5, pp. 994-1007, 2011.

[60] M. H. Terra, J. Y. Ishihara, and G. Jesus, "Information filtering and array algorithms for discrete-time Markovian jump linear systems," IEEE Transactions on Automatic Control, vol. 54, no. 1, pp. 158-162, 2009.

[61] L. Wu, P. Shi, H. Gao, and C. Wang, " $H_{\infty}$ filtering for 2D Markovian jump systems," Automatica, vol. 44, no. 7, pp. 1849-1858, 2008.

[62] J. Xiong and J. Lam, "Fixed-order robust $H_{\infty}$ filter design for Markovian jump systems with uncertain switching probabilities," IEEE Transactions on Signal Processing, vol. 54, no. 4, pp. 14211430, 2006.

[63] L. Zhang, " $H_{\infty}$ estimation for discrete-time piecewise homogeneous Markov jump linear systems," Automatica, vol. 45, no. 11, pp. 2570-2576, 2009.

[64] X. Zhang and Y. Zheng, "Nonlinear $H_{\infty}$ filtering for interconnected Markovian jump systems," Journal of Systems Engineering and Electronics, vol. 17, no. 1, pp. 138-146, 2006.

[65] L. Wu, P. Shi, and H. Gao, "State estimation and sliding-mode control of Markovian jump singular systems," IEEE Transactions on Automatic Control, vol. 55, no. 5, pp. 1213-1219, 2010.

[66] J. Zhang, Y. Xia, and E. K. Boukas, "New approach to $H_{\infty}$ control for Markovian jump singular systems," IET Control Theory and Applications, vol. 4, no. 11, pp. 2273-2284, 2010.

[67] X.He, Z. Wang, and D. H. Zhou, "Robust fault detection for networked systems with communication delay and data missing," Automatica, vol. 45, no. 11, pp. 2634-2639, 2009.

[68] M. Zhong, H. Ye, P. Shi, and G. Wang, "Fault detection for Markovian jump systems," Proceedings of the Institution of Electrical Engineers, vol. 152, no. 4, pp. 397-402, 2005.

[69] L. Zhang, E.-K. Boukas, and J. Lam, "Analysis and synthesis of Markov jump linear systems with time-varying delays and partially known transition probabilities," IEEE Transactions on Automatic Control, vol. 53, no. 10, pp. 2458-2464, 2008.

[70] Y. Zhao, L. Zhang, S. Shen, and H. Gao, "Robust stability criterion for discrete-time uncertain markovian jumping neural networks with defective statistics of modes transitions," IEEE Transactions on Neural Networks, vol. 22, no. 1, pp. 164-170, 2011. 
[71] S. X. Ding, T. Jeinsch, P. M. Frank, and E. L. Ding, "A unified approach to the optimization of fault detection systems," International Journal of Adaptive Control and Signal Processing, vol. 14, no. 7, pp. 725-745, 2000.

[72] Y. Zheng, H. Fang, and H. O. Wang, "Takagi-Sugeno fuzzy-model-based fault detection for networked control systems with Markov delays," IEEE Transactions on Systems, Man, and Cybernetics B, vol. 36, no. 4, pp. 924-929, 2006.

[73] M. Zhong, S. X. Ding, and E. L. Ding, "Optimal fault detection for linear discrete time-varying systems," Automatica, vol. 46, no. 8, pp. 1395-1400, 2010.

[74] Z. Wang, Y. Liu, and X. Liu, " $H_{\infty}$ filtering for uncertain stochastic time-delay systems with sectorbounded nonlinearities," Automatica, vol. 44, no. 5, pp. 1268-1277, 2008.

[75] G. Wei, Z. Wang, H. Shu, and J. Fang, "A delay-dependent approach to $H_{\infty}$ filtering for stochastic delayed jumping systems with sensor non-linearities," International Journal of Control, vol. 80, no. 6, pp. 885-897, 2007.

[76] X. Jiang and Q. L. Han, “On designing fuzzy controllers for a class of nonlinear networked control systems," IEEE Transactions on Fuzzy Systems, vol. 16, no. 4, pp. 1050-1058, 2008.

[77] Z. Wang, D. W. C. Ho, and X. Liu, "A note on the robust stability of uncertain stochastic fuzzy systems with time-delays," IEEE Transactions on Systems, Man, and Cybernetics A, vol. 34, no. 4, pp. 570-576, 2004.

[78] W. Zhang, B.-S. Chen, and C.-S. Tseng, "Robust $H_{\infty}$ filtering for nonlinear stochastic systems," IEEE Transactions on Signal Processing, vol. 53, no. 2, pp. 589-598, 2005.

[79] F. S. Cattivelli and A. H. Sayed, "Diffusion strategies for distributed Kalman filtering and smoothing," IEEE Transactions on Automatic Control, vol. 55, no. 9, pp. 1520-1520, 2010.

[80] L. Chai, B. Hu, and P. Jiang, "Distributed state estimation based on quantized observations in a bandwidth constrained sensor network," in Proceedings of the 7th World Congress on Intelligent Control and Automation (WCICA '08), pp. 2411-2415, June 2008.

[81] M. Farina, G. Ferrari-Trecate, and R. Scattolini, “Distributed moving horizon estimation for sensor networks," in Proceedings of the 1st IFAC Workshop on Estimation and Control of Networked Systems (NecSys '09), pp. 126-131, Venice, Italy, September 2009.

[82] M. Kamgarpour and C. Tomlin, "Convergence properties of a decentralized Kalman filter," in Proceedings of the 47th IEEE Conference on Decision and Control (CDC '08), pp. 3205-3210, Cancun, Mexico, December 2008.

[83] R. Olfati-Saber, "Kalman-consensus filter: optimality, stability, and performance," in Proceedings of the 48th IEEE Conference on Decision and Control held Jointly with 28th Chinese Control Conference (CDC/CCC '09), pp. 7036-7042, New Orleans, La, USA, December 2009.

[84] R. Olfati-Saber, "Flocking for multi-agent dynamic systems: algorithms and theory," IEEE Transactions on Automatic Control, vol. 51, no. 3, pp. 401-420, 2006.

[85] B. Shen, Z. Wang, and X. Liu, "A stochastic sampled-data approach to distributed $H_{\infty}$ filtering in sensor networks," IEEE Transactions on Circuits and Systems I, vol. 58, no. 9, pp. 2237-2246, 2011.

[86] B. Shen, Z. Wang, and Y. S. Hung, "Distributed consensus $H_{\infty}$ filtering in sensor networks with multiple missing measurements: the finite-horizon case," Automatica, vol. 46, no. 10, pp. 1682-1688, 2010.

[87] B. Shen, Z. Wang, Y. S. Hung, and G. Chesi, "Distributed $H_{\infty}$ filtering for polynomial nonlinear stochastic systems in sensor networks," IEEE Transactions on Industrial Electronics, vol. 58, no. 5, pp. 1971-1979, 2011.

[88] W. Yu, G. Chen, Z. Wang, and W. Yang, "Distributed consensus filtering in sensor networks," IEEE Transactions on Systems, Man, and Cybernetics B, vol. 39, no. 6, pp. 1568-1577, 2009.

[89] H. Zhang, G. Feng, G. Duan, and X. Lu, "H $H_{\infty}$ filtering for multiple-time-delay measurements," IEEE Transactions on Signal Processing, vol. 54, no. 5, pp. 1681-1688, 2006.

[90] F. S. Cattivelli and A. H. Sayed, "Diffusion strategies for distributed Kalman filtering: formulation and performance analysis," in Proceedings of the Cognitive Information Processing, pp. 36-41, Santorini, Greece, 2008.

[91] R. Carli, A. Chiuso, L. Schenato, and S. Zampieri, “Distributed Kalman filtering based on consensus strategies," IEEE Journal on Selected Areas in Communications, vol. 26, no. 4, pp. 622-633, 2008.

[92] R. Olfati-Saber, "Distributed Kalman filtering for sensor networks," in Proceedings of the 46th IEEE Conference on Decision and Control (CDC'07), pp. 5492-5498, New Orleans, La, USA, December 2007.

[93] R. Olfati-Saber and J. S. Shamma, "Consensus filters for sensor networks and distributed sensor fusion," in Proceedings of the 44th IEEE Conference on Decision and Control, and the European Control Conference (CDC-ECC '05), pp. 6698-6703, Seville, Spain, December 2005. 
[94] V. A. Ugrinovskii, "Distributed robust filtering with $H_{\infty}$ consensus of estimates," in Proceedings of the American Control Conference (ACC '10), pp. 1374-1379, July 2010.

[95] H. Dong, Z. Wang, and H. Gao, " $H_{\infty}$ filtering for systems with repeated scalar nonlinearities under unreliable communication links," Signal Processing, vol. 89, no. 8, pp. 1567-1575, 2009.

[96] H. Dong, Z. Wang, and H. Gao, “Observer-based $H_{\infty}$ control for systems with repeated scalar nonlinearities and multiple packet losses," International Journal of Robust and Nonlinear Control, vol. 20, no. 12, pp. 1363-1378, 2010.

[97] H. Dong, Z. Wang, and H. Gao, "Robust $H_{\infty}$ filtering for a class of nonlinear networked systems with multiple stochastic communication delays and packet dropouts," IEEE Transactions on Signal Processing, vol. 58, no. 4, pp. 1957-1966, 2010.

[98] H. Dong, Z. Wang, D. W. C. Ho, and H. Gao, "Robust $H_{\infty}$ fuzzy output-feedback control with multiple probabilistic delays and multiple missing measurements," IEEE Transactions on Fuzzy Systems, vol. 18, no. 4, pp. 712-725, 2010.

[99] Z. Wang, D. W. C. Ho, H. Dong, and H. Gao, "Robust $H_{\infty}$ finite-horizon control for a class of stochastic nonlinear time-varying systems subject to sensor and actuator saturations," IEEE Transactions on Automatic Control, vol. 55, no. 7, pp. 1716-1722, 2010.

[100] H. Dong, Z. Wang, and H. Gao, "Fault detection for Markovian jump systems with sensor saturations and randomly varying nonlinearities," IEEE Transactions on Circuits and Systems I, vol. 59, no. 10, pp. 2354-2362, 2012.

[101] H. Dong, Z. Wang, J. Lam, and H. Gao, "Fuzzy-model-based robust fault detection with stochastic mixed time-delays and successive packet dropouts," IEEE Transactions on Systems, Man, and Cybernetics B, vol. 42, no. 2, pp. 365-376, 2012.

[102] H. Dong, J. Lam, and H. Gao, "Distributed $H_{\infty}$ filtering for repeated scalar nonlinear systems with random packet losses in sensor networks," International Journal of Systems Science, vol. 42, no. 9, pp. 1507-1519, 2011.

[103] H. Dong, Z. Wang, and H. Gao, “Distributed filtering for a class of time-varying systems over sensor networks with quantization errors and successive packet dropouts," IEEE Transactions on Signal Processing, vol. 60, no. 6, pp. 3164-3173, 2012.

[104] H. Dong, Z. Wang, and H. Gao, "Distributed $H_{\infty}$ filtering for a class of Markovian jump nonlinear time-delay systems over lossy sensor networks," IEEE Transactions on Industrial Electronics. In press.

[105] D. Ding, Z. Wang, H. Dong, and H. Shu, "State estimation with stochastic parameters and nonlinearities through sensor networks: the finite-horizon case," Automatica, vol. 48, no. 8, pp. 1575$1585,2012$. 


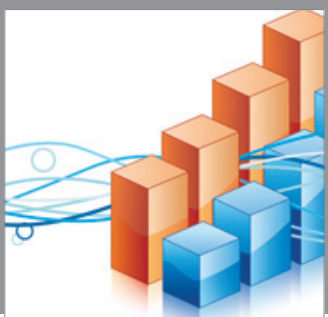

Advances in

Operations Research

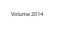

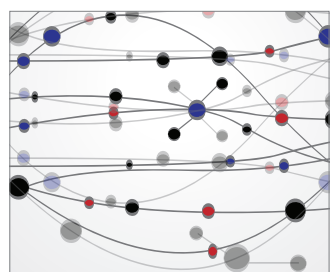

\section{The Scientific} World Journal
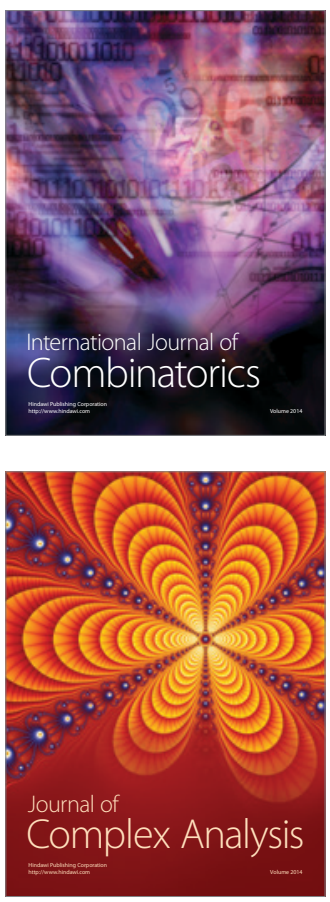

International Journal of

Mathematics and

Mathematical

Sciences
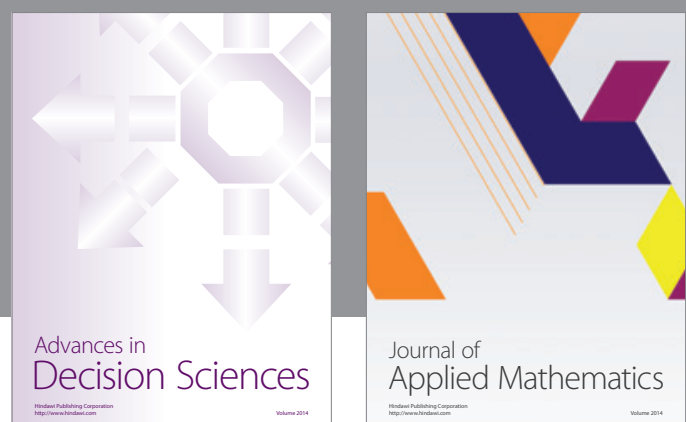

Journal of

Applied Mathematics
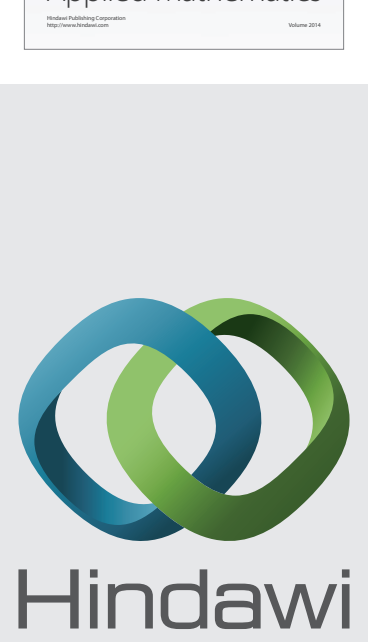

Submit your manuscripts at http://www.hindawi.com
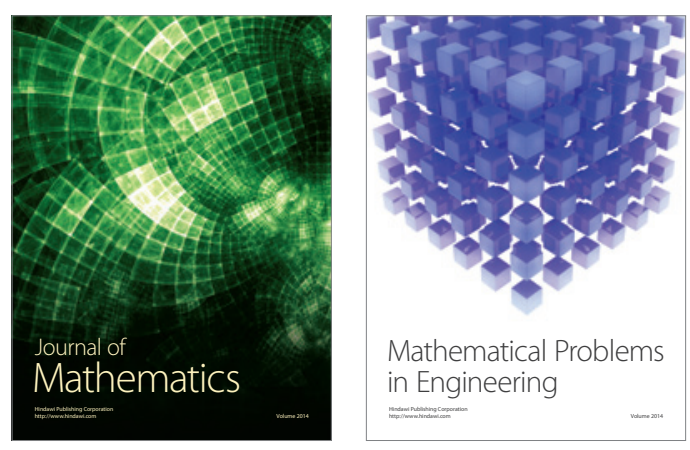

Mathematical Problems in Engineering
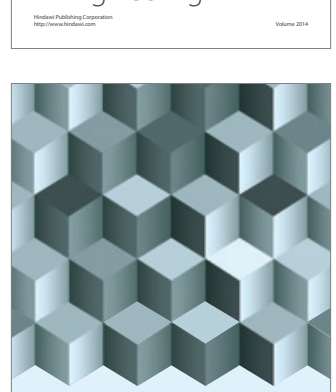

Journal of

Function Spaces
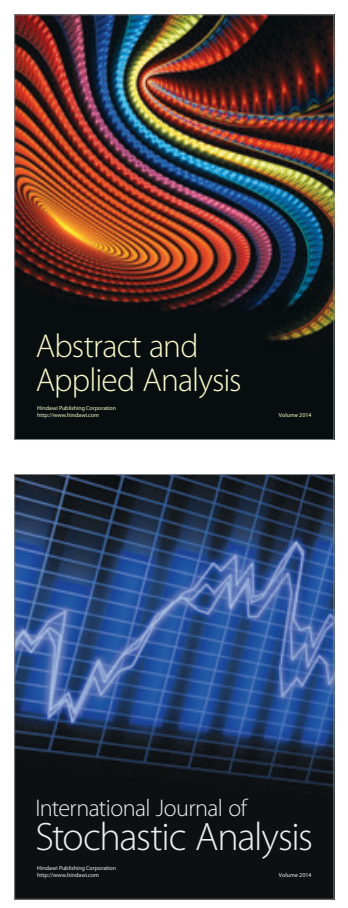

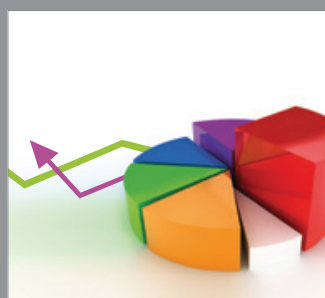

ournal of

Probability and Statistics

Promensencen
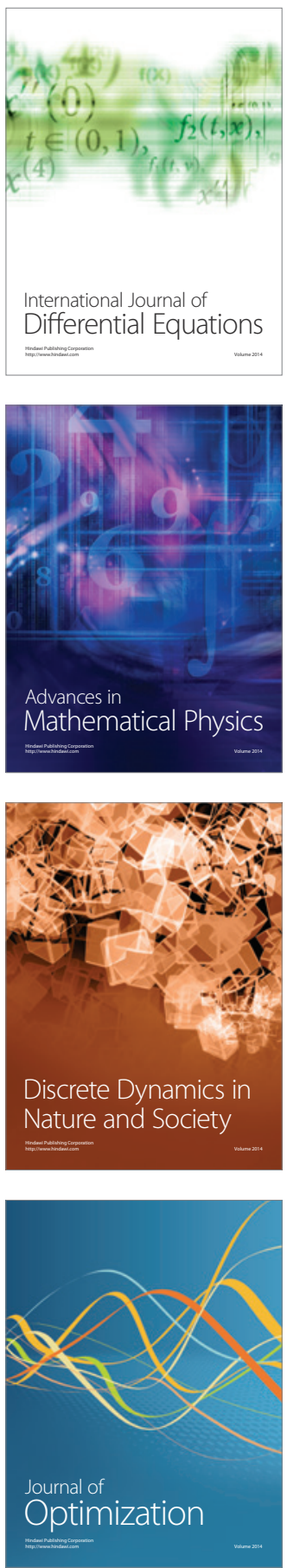